\title{
An Interactive Fuzzy Satisficing Method for Multiobjective Linear Programming Problems with Random Fuzzy Variables Using Possibility-based Probability Model
}

\author{
Masatoshi Sakawa*, Takeshi Matsui, Hideki Katagiri \\ Faculty of Engineering, Hiroshima University, Higashi-Hiroshima, 739-8527, Hiroshima, Japan \\ *Corresponding Author: sakawa@hiroshima-u.ac.jp
}

Copyright @2014 Horizon Research Publishing All rights reserved.

\begin{abstract}
This paper formulates multiobjective linear programming problems where each coefficient of the objective functions is expressed by a random fuzzy variable. Assuming that the decision maker concerns about the probability that each of the objective function values is smaller than or equal to a certain target value, the fuzzy goals of the decision maker for the probabilities are introduced. Then, the possibility-based probability model to maximize the degrees of possibility with respect to the attained probability is considered. For solving transformed deterministic problems efficiently, particle swarm optimization for nonlinear programming problems is introduced. An interactive fuzzy satisficing method is presented for deriving a satisficing solution for a decision maker efficiently by updating the reference probability levels. An illustrative numerical example is provided to demonstrate the feasibility and efficiency of the proposed method.
\end{abstract}

Keywords Multiobjective linear programming, Random fuzzy programming, Possibility, Probability maximization, Interactive method

\section{Introduction}

In actual decision making situations, we must often make a decision on the basis of vague information or uncertain data. For such decision making problems involving uncertainty, there exist two typical approaches: probability-theoretic approach [1-4] and fuzzy-theoretic one [5-7]. Stochastic programming, as an optimization method based on the probability theory, have been developing in various ways including two stage problems considered by Dantzig [8] and chance constrained programming proposed by Charnes et al. [9].

In most practical situations, however, it is natural to consider that the uncertainty in real world decision making problems is often expressed by a fusion of fuzziness and randomness rather than either fuzziness or random- ness. For handling not only the decision maker's vague judgments in multiobjective problems but also the randomness of the parameters involved in the objectives and/or constraints, Sakawa and his colleagues incorporated their interactive fuzzy satisficing methods for deterministic problems $[5,10]$ into multiobjective stochastic programming problems, through the introduction of several stochastic programming models such as expectation optimization $[11,12]$, variance minimization [11], probability maximization $[11,13,14]$ and fractile criterion optimization [11], to derive a satisficing solution for a decision maker from Pareto optimal solution sets.

In multiobjective stochastic programming problems, it is implicitly assumed that uncertain parameters or coefficients can be expressed as random variables in probability theory. This means that the realized values of random parameters under the occurrence of some event are assumed to be definitely represented with real values.

However, it is natural to consider that the possible realized values of these random parameters are often only ambiguously known to the experts. In this case, it may be more appropriate to interpret the experts' ambiguous understanding of the realized values of random parameters under the occurrence of events as fuzzy numbers. From such a point of view, a fuzzy random variable was first introduced by Kwakernaak [15], and its mathematical basis was constructed by Puri and Ralescu [16]. Studies on linear programming problems with fuzzy random variable coefficients, called fuzzy random linear programming problems, were initiated by Wang and Qiao [17] and Qiao, Zhang and Wang [18] as seeking the probability distribution of the optimal solution and optimal value.

On the other hand, from a viewpoint of ambiguity and randomness different from fuzzy random variables [15-17], by considering the experts' ambiguous understanding of means and variances of random variables, a concept of random fuzzy variables was proposed, and mathematical programming problems with random fuzzy variables were formulated together with the development of a simulation-based approximate solution 
method [19].

A recently published book of Sakawa et al. [20] is devoted to introducing the latest advances in the field of multiobjective optimization under both fuzziness and randomness on the basis of authors' continuing research works. Special stress is placed on interactive decision making aspects of fuzzy stochastic multiobjective programming for human-centered systems under uncertainty in most realistic situations when dealing with both fuzziness and randomness.

Under these circumstances, in this paper, we consider multiobjective linear programming problems involving random fuzzy variables. To deal with the formulated random fuzzy multiobjective linear programming problems, we assume that the decision maker concerns about the probabilities that each of the objective function values is smaller than or equal to a certain target value. By considering the imprecise nature of the human judgments, we introduce the fuzzy goals of the decision maker for the probabilities. Assuming that the decision maker is willing to maximize the degrees of possibility with respect to the attained probability, we consider the possibility-based probability model for random fuzzy multiobjective linear programming problems. For solving transformed deterministic problems efficiently, particle swarm optimization for nonlinear programming problems [22] is introduced. Then, we present an interactive fuzzy satisficing method to derive a satisficing solution for the decision maker by updating the reference possibility levels. An illustrative numerical example is provided to demonstrate the feasibility and efficiency of the proposed method.

\section{Random fuzzy variables}

In the framework of stochastic programming, it is implicitly assumed that the uncertain parameter which well represents the stochastic factor of real systems can be definitely expressed as a single random variable. This means that the realized values of random parameters under the occurrence of some event are assumed to be definitely represented with real values.

Depending on the situations, however, it is natural to consider that the possible realized values of these random parameters are often only ambiguously known to the experts. In this case, it may be more appropriate to interpret the experts' ambiguous understanding of the realized values of random parameters as fuzzy numbers. From such a point of view, a fuzzy random variable was first introduced by Kwakernaak [15], and its mathematical basis was constructed by Puri and Ralescu [16].

From the expert's experimental point of view, however, the experts may think of a collection of random variables to be appropriate to express stochastic factors rather than only a single random variables. In this case, reflecting the expert's conviction degree that each of random variables properly represents the stochastic factor, it would be quite reasonable to assign the different degrees of possibility to each of random variables. For handling such an uncertain parameter, a random fuzzy variable was defined by Liu [19] as a function from a possibility space to a collection of random variables, which is considered to be an extended concept of fuzzy variable [21]. It should be noted here that the fuzzy variables can be viewed as another way of dealing with the imprecision which was originally represented by fuzzy sets. Although we can employ Liu's definition, for consistently discussing various concepts in relation to the fuzzy sets, we define the random fuzzy variables by extending not the fuzzy variables but the fuzzy sets.

Definition 1 (Random fuzzy variable) Let $\Gamma$ be a collection of random variables. Then, a random fuzzy variable $\overline{\tilde{C}}$ is defined by its membership function

$$
\mu_{\tilde{\tilde{C}}}: \Gamma \rightarrow[0,1]
$$

In Definition 1, the membership function $\mu_{\tilde{\tilde{C}}}$ assigns each random variable $\bar{\gamma} \in \Gamma$ to a real number $\mu_{\tilde{\tilde{C}}}(\bar{\gamma})$. It should be noted here that if $\Gamma$ is defined as $\mathbb{R}$, then (1) becomes equivalent to the membership function of an ordinary fuzzy set. In this sense, a random fuzzy variable can be regarded as an extended concept of fuzzy sets. On the other hand, if $\Gamma$ is defined as a singleton $\Gamma=\{\bar{\gamma}\}$ and $\mu_{\tilde{C}_{\overline{\widetilde{C}}}}(\bar{\gamma})=1$, then the corresponding random fuzzy variable $\overline{\tilde{C}}$ can be viewed as an ordinary random variable.

When taking account of the imprecise nature of the realized values of random variables, it would be appropriate to employ the concept of fuzzy random variables. However, it should be emphasized here that if mean and/or variance of random variables are specified by the expert as a set of real values or fuzzy sets, such uncertain parameters can be represented by not fuzzy random variables but random fuzzy variables.

As a simple example of random fuzzy variables, we consider a Gaussian random variable whose mean value is not definitely specified as a constant. For example, when some random parameter $\bar{\gamma}$ is represented by the Gaussian random variable $N\left(s_{i}, 10^{2}\right)$ where the expert identifies a set $\left\{s_{1}, s_{2}, s_{3}\right\}$ of possible mean values as $\left(s_{1}, s_{2}, s_{3}\right)=(90,100,110)$, if the membership function $\mu_{\tilde{\tilde{C}}}$ is defined by

$$
\mu_{\tilde{\tilde{C}}}(\bar{\gamma})= \begin{cases}0.5 & \text { if } \bar{\gamma} \sim N\left(90,10^{2}\right) \\ 0.7 & \text { if } \bar{\gamma} \sim N\left(100,10^{2}\right) \\ 0.3 & \text { if } \bar{\gamma} \sim N\left(110,10^{2}\right) \\ 0 & \text { otherwise, }\end{cases}
$$

then $\overline{\tilde{C}}$ is a random fuzzy variable. More generally, when the mean values are expressed as fuzzy sets or fuzzy numbers, the corresponding random variable with the fuzzy mean is represented by a random fuzzy variable.

\section{Problem formulation}

In the remainder of this paper, considering the possible realized values of random parameters are often only ambiguously known to the experts, we focus on multiobjective linear programming problems with random fuzzy variables. For that purpose, we formulate the random fuzzy multiobjective linear programming problems 
expresses by

$$
\left.\begin{array}{rl}
\operatorname{minimize} & \overline{\tilde{\boldsymbol{C}}}_{1} \boldsymbol{x} \\
& \cdots \\
\text { minimize } & \overline{\tilde{\boldsymbol{C}}}_{k} \boldsymbol{x} \\
\text { subject to } & A \boldsymbol{x} \leq \boldsymbol{b}, \boldsymbol{x} \geq \mathbf{0}
\end{array}\right\}
$$

where $\boldsymbol{x}=\left(x_{1}, \ldots, x_{n}\right)^{T}$ is an $n$-dimensional decision variable column vector, $A$ is an $m \times n$ matrix, $\boldsymbol{b}$ is an $m \times 1$ constant column vector, and $\overline{\tilde{C}}_{l}=\left(\overline{\tilde{C}}_{l 1}, \ldots, \overline{\tilde{C}}_{l n}\right)$ is a random fuzzy variable coefficient vector. Here, assume that $\overline{\tilde{C}}_{l j}$ is a Gaussian random variable whose mean value is a fuzzy number $\tilde{M}_{l j}$ characterized by the membership function

$$
\mu_{\tilde{M}_{l j}}(t)=\left\{\begin{array}{cl}
L\left(\frac{m_{l j}-t}{\alpha_{l j}}\right) & \text { if } m_{l j} \geq t \\
R\left(\frac{t-m_{l j}}{\beta_{l j}}\right) & \text { if } m_{l j}<t,
\end{array}\right.
$$

where the shape functions $L$ and $R$ are nonincreasing continuous functions from $[0, \infty)$ to $[0,1], m_{l j}$ is the mean value, and $\alpha_{l j}$ and $\beta_{l j}$ are positive numbers which represent left and right spreads. Fig. 1 illustrates an example of the membership function $\mu_{\tilde{M}_{l j}}(\tau)$.

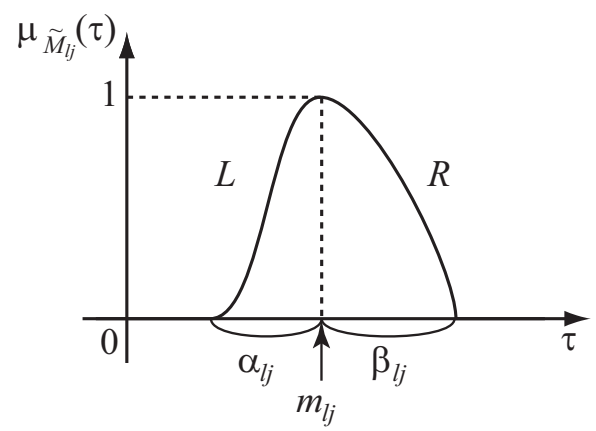

Figure 1. An example of the membership function $\mu_{\tilde{M}_{l j}}(\tau)$.

Let $\Gamma$ be a collection of all possible Gaussian random variables $N\left(s, \sigma^{2}\right)$ where $s \in(-\infty, \infty)$ and $\sigma^{2} \in(0, \infty)$. Then, the membership function of $\overline{\tilde{C}}_{l j}$ is expressed as

$$
\mu_{\overline{\tilde{C}}_{l j}}\left(\bar{\gamma}_{l j}\right)=\left\{\mu_{\tilde{M}_{l j}}\left(s_{l j}\right) \mid \bar{\gamma}_{l j} \sim N\left(s_{l j}, \sigma_{l j}^{2}\right)\right\}, \forall \bar{\gamma}_{l j} \in \Gamma .
$$

Using the Zadeh's extension principle, each objective function $\overline{\tilde{C}}_{l} \boldsymbol{x}$ is expressed as a random fuzzy variable characterized by the membership function

$$
\begin{aligned}
& \mu_{\tilde{\boldsymbol{C}}_{l} \boldsymbol{x}}\left(\overline{\boldsymbol{u}}_{l}\right) \sup _{\overline{\boldsymbol{\gamma}}_{l}}\left\{\min _{1 \leq j \leq n} \mu_{\overline{\tilde{C}}_{l j}}\left(\bar{\gamma}_{l j}\right) \mid \bar{u}_{l}=\sum_{j=1}^{n} \bar{\gamma}_{l j} x_{j}\right\}, \\
& \forall \bar{u}_{l} \in \Gamma,
\end{aligned}
$$

where $\bar{\gamma}_{l}=\left(\bar{\gamma}_{1}, \ldots, \bar{\gamma}_{n}\right)$.

By substituting (4) into (5), the membership function of a random fuzzy variable corresponding to the objective function $\overline{\tilde{\boldsymbol{C}}_{l}} \boldsymbol{x}$ in (2) is rewritten as

$$
\begin{aligned}
\mu_{\overline{\tilde{C}}_{l} \boldsymbol{x}}\left(\bar{u}_{l}\right) & \sup _{\boldsymbol{s}_{l}}\left\{\min _{1 \leq j \leq n} \mu_{\tilde{M}_{l j}}\left(s_{l j}\right) \mid \bar{u}_{l} \sim N\left(\sum_{j=1}^{n} s_{l j} x_{j}, \sum_{j=1}^{n} \sigma_{l j}^{2} x_{j}^{2}\right)\right\},
\end{aligned}
$$

where $s_{l}=\left(s_{l 1}, \ldots, s_{l n}\right)$.

Observing $\overline{\tilde{C}}_{l} \boldsymbol{x}$ is expressed as a random fuzzy variable with the membership function $\mu_{\overline{\tilde{C}} \boldsymbol{x}}$ defined by (6), it is significant to realize that the fuzzy random programming models cannot be applied.

\section{Possibility-based probability model}

Assuming that the decision maker (DM) concerns about the probability that each of the objective function values $\overline{\tilde{C}}_{l} \boldsymbol{x}$ is smaller than or equal to a certain target values $f_{l}$, we introduce the probability $P\left(\omega \mid \tilde{\boldsymbol{C}}_{l}(\omega) \boldsymbol{x} \leq f_{l}\right)$ which is expressed as a fuzzy set $\tilde{P}_{l}$ with the membership function

$$
\mu_{\tilde{P}_{l}}\left(p_{l}\right)=\sup _{\bar{u}_{l}}\left\{\mu_{\tilde{\boldsymbol{C}}_{l} \boldsymbol{x}}\left(\bar{u}_{l}\right) \mid p_{l}=P\left(\omega \mid u_{l}(\omega) \leq f_{l}\right)\right\},
$$

where $f_{l}, l=1, \ldots, k$ are target values specified by the DM as constants.

Considering the imprecise nature of the DM's judgments for the probabilities $\tilde{P}_{l}$ with respect to the random fuzzy objective function values $\overline{\tilde{\boldsymbol{C}}}_{l} \boldsymbol{x}, l=1, \ldots, k$, we introduce the fuzzy goals $\tilde{G}_{l}, l=1, \ldots, k$ such as " $\tilde{P}_{l}$ should be greater than or equal to a certain value." Such fuzzy goals $\tilde{G}_{l}, l=1, \ldots, k$ can be quantified by eliciting corresponding membership functions

$$
\mu_{\tilde{G}_{l}}(p)=\left\{\begin{array}{lll}
0 & \text { if } \quad p \leq p_{l}^{0} \\
g_{l}(p) & \text { if } \quad p_{l}^{0} \leq p \leq p_{l}^{1}, \quad l=1, \ldots, k \\
1 & \text { if } \quad p_{l}^{1} \leq p,
\end{array}\right.
$$

where $g_{l}(p), l=1, \ldots, k$ are nondecreasing functions. Fig. 2 illustrates a possible shape of the membership function for the fuzzy goal $\tilde{G}_{l}$.

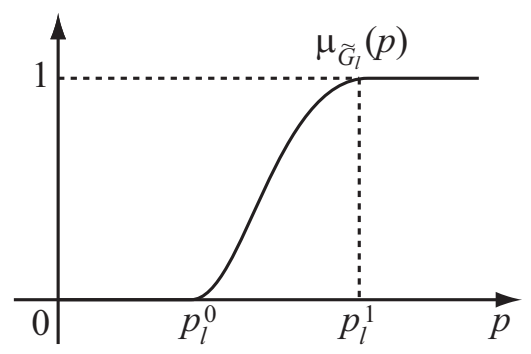

Figure 2. An example of a membership function $\mu_{\tilde{G}_{l}}(p)$ of a fuzzy goal $\tilde{G}_{l}$

Recalling that the membership function is regarded as a possibility distribution, the degree of possibility that the probability $\tilde{P}_{l}$ attains the fuzzy goal $\tilde{G}_{l}$ is expressed as

$$
\Pi_{\tilde{P}_{l}}\left(\tilde{G}_{l}\right) \triangleq \sup _{p_{l}} \min \left\{\mu_{\tilde{P}_{l}}\left(p_{l}\right), \mu_{\tilde{G}_{l}}\left(p_{l}\right)\right\} \quad l=1, \ldots, k .
$$

Fig. 3 illustrates the degree of possibility $\Pi_{\tilde{P}_{l}}\left(\tilde{G}_{l}\right)$.

Now, assuming that the DM is willing to maximize the degrees of possibility with respect to the attained 


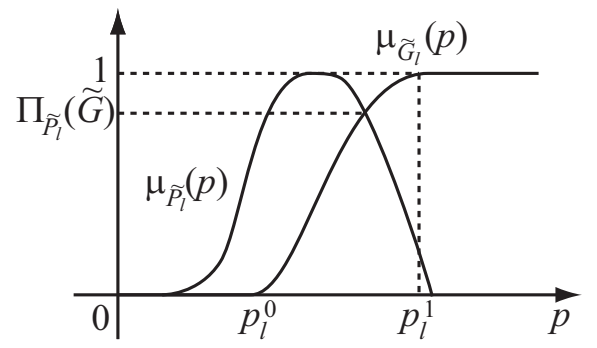

Figure 3. The degree of possibility $\Pi_{\tilde{P}_{l}}\left(\tilde{G}_{l}\right)$

probability, we consider the possibility-based probability model for multiobjective random fuzzy integer programming problems formulated as

$$
\left.\begin{array}{cl}
\operatorname{maximize} & \Pi_{\tilde{P}_{1}}\left(\tilde{G}_{1}\right) \\
& \cdots \cdots \\
\text { maximize } & \Pi_{\tilde{P}_{k}}\left(\tilde{G}_{k}\right) \\
\text { subject to } & A \boldsymbol{x} \leq \boldsymbol{b}, \boldsymbol{x} \geq \mathbf{0}
\end{array}\right\}
$$

or equivalently

$$
\left.\begin{array}{rl}
\text { maximize } & h_{1} \\
& \vdots \\
\text { maximize } & h_{k} \\
\text { subject to } & \Pi_{\tilde{P}_{l}}\left(\tilde{G}_{l}\right) \geq h_{l}, l=1, \ldots, k \\
& A \boldsymbol{x} \leq \boldsymbol{b}, \boldsymbol{x} \geq \mathbf{0}
\end{array}\right\}
$$

\section{Interactive fuzzy satisficing method}

Observing that (11) can be regarded as a multiobjective programming problem, a complete optimal solution that simultaneously minimizes all of the objective functions does not always exist when the objective functions conflict with each other. Thus, instead of a complete optimal solution, a solution concept of Pareto optimality plays an important role in multiobjective programming [5].

To be more specific, in the general form of multiobjective programming problem

$$
\left.\begin{array}{ll}
\operatorname{minimize} & z_{1}(\boldsymbol{x}) \\
& \cdots \cdots \\
\text { minimize } & z_{k}(\boldsymbol{x}) \\
\text { subject to } & \boldsymbol{x} \in X
\end{array}\right\}
$$

where $X$ denotes the set of all feasible solutions, since there does not always exist a solution minimizing all of the objective functions simultaneously, the solution concept of Pareto optimality plays an important role and it is defined as follows.

Definition 2 (Pareto optimal solution) $A$ point $\boldsymbol{x}^{*} \in X$ is said to be a Pareto optimal solution if and only if there does not exist another $\boldsymbol{x} \in X$ such that $z_{i}(\boldsymbol{x}) \leq z_{i}\left(\boldsymbol{x}^{*}\right)$ for all $i \in\{1, \ldots, k\}$ and $z_{j}(\boldsymbol{x}) \neq z_{j}\left(\boldsymbol{x}^{*}\right)$ for at least one $j \in\{1, \ldots, k\}$.

As can be seen from Definition 2, in general there exist an infinite number of Pareto optimal solutions if the feasible region $X$ is not empty. In real-world decision making problems, to make a reasonable decision or implement a desirable scheme, the decision maker should select one point from among the set of Pareto optimal solutions [5].

For the multiobjective programming problem (11) under consideration, in order to generate a candidate for the satisficing solution which is also Pareto optimal, the DM is asked to specify the reference levels of $h_{l}, l=1, \ldots, k$, called reference possibility levels. For the reference levels $\hat{h}_{l}, l=1, \ldots, k$ specified by the DM, the corresponding Pareto optimal solution, which is, in the minimax sense, nearest ito the requirement or better than that if the reference possibility levels are attainable, is obtained by solving the augmented minimax problem [5]

$$
\left.\begin{array}{rl}
\operatorname{minimize} & \max _{l=1, \ldots, k}\left\{\hat{h}_{l}-h_{l}+\rho \sum_{l=1}^{k}\left(\hat{h}_{l}-h_{l}\right)\right\} \\
\text { subject to } & \Pi_{\tilde{P}_{l}}\left(\tilde{G}_{l}\right) \geq h_{l}, l=1, \ldots, k \\
& A \boldsymbol{x} \leq \boldsymbol{b}, \boldsymbol{x} \geq \mathbf{0}
\end{array}\right\}
$$

where $\rho$ is a sufficiently small positive number.

It should be noted here that (13) involves the possibility constraints $\Pi_{\tilde{P}_{l}}\left(\tilde{G}_{l}\right) \geq h_{l}, l=1, \ldots, k$, solution methods for ordinary mathematical programming problems cannot be directly applied. Realizing such difficulty, we deal with the possibility constraints in the following way.

From (9), the possibility constraints $\Pi_{\tilde{P}_{l}}\left(\tilde{G}_{l}\right) \geq h_{l}$ in (11) or (13) are equivalently replaced by the conditions that there exists a $p_{l}$ such that $\mu_{\tilde{P}_{l}}\left(p_{l}\right) \geq h_{l}$ and $\mu_{\tilde{G}_{l}}(p) \geq h_{l}$, namely,

$$
\begin{array}{r}
\sup _{\boldsymbol{s}_{l}} \min _{1 \leq j \leq n}\left\{\mu_{\tilde{M}_{l j}}\left(s_{l j}\right) \mid p_{l}=P\left(\omega \mid u_{l}(\omega) \leq f_{l}\right),\right. \\
\left.\bar{u}_{l} \sim N\left(\sum_{j=1}^{n} s_{l j} x_{j}, \sum_{j=1}^{n} \sigma_{l j}^{2} x_{j}^{2}\right)\right\} \geq h_{l}
\end{array}
$$

and $p_{l} \geq \mu_{\tilde{G}_{l}}^{\star}\left(h_{l}\right)$, where $\boldsymbol{s}_{l}=\left(s_{l 1}, \ldots, s_{l n}\right)$ and $\mu_{\tilde{G}_{l}}^{\star}\left(h_{l}\right)$ is a pseudo inverse function defined as $\mu_{\tilde{G}_{l}}^{\star}\left(h_{l}\right)=$ $\sup \left\{p_{l} \mid \mu_{\tilde{G}_{l}}\left(p_{l}\right) \geq h_{l}\right\}$. This implies that there exists a vector $\left(p_{l}, \boldsymbol{s}_{l}, \bar{u}_{l}\right)$ such that

$$
\begin{gathered}
\min _{1 \leq j \leq n} \mu_{\tilde{M}_{l j}}\left(s_{l j}\right) \geq h_{l}, \bar{u}_{l} \sim N\left(\sum_{j=1}^{n} s_{l j} x_{j}, \sum_{j=1}^{n} \sigma_{l j}^{2} x_{j}^{2}\right), \\
p_{l}=P\left(\omega \mid u_{l}(\omega) \leq f_{l}\right), p_{l} \geq \mu_{\tilde{G}_{l}}^{\star}\left(h_{l}\right),
\end{gathered}
$$

which can be equivalently transformed into the condition that there exists a vector $\left(s_{l}, \bar{u}_{l}\right)$ such that

$$
\begin{gathered}
\mu_{\tilde{M}_{l j}}\left(s_{l j}\right) \geq h_{l}, j=1, \ldots, n, \\
\bar{u}_{l} \sim N\left(\sum_{j=1}^{n} s_{l j} x_{j}, \sum_{j=1}^{n} \sigma_{l j}^{2} x_{j}^{2}\right), \\
P\left(\omega \mid u_{l}(\omega) \leq f_{l}\right) \geq \mu_{\tilde{G}_{l}}^{\star}\left(h_{l}\right) .
\end{gathered}
$$

In view of $(3)$, it follows that

$\mu_{\tilde{M}_{l j}}\left(s_{l j}\right) \geq h_{l} \Leftrightarrow s_{l j} \in\left[m_{l j}-L^{\star}\left(h_{l}\right) \alpha_{l j}, m_{l j}+R^{\star}\left(h_{l}\right) \beta_{l j}\right]$, 
where $L^{\star}\left(h_{l}\right)$ and $R^{\star}\left(h_{l}\right)$ are pseudo inverse functions defined as $L^{\star}\left(h_{l}\right)=\sup \left\{t \mid L(t) \geq h_{l}\right\}$ and $R^{\star}\left(h_{l}\right)=$ $\sup \left\{t \mid R(t) \geq h_{l}\right\}$. Hence, (15) is rewritten as the equivalent condition that there exists a $\bar{u}_{l}$ such that

$$
\begin{gathered}
P\left(\omega \mid u_{l}(\omega) \leq f_{l}\right) \geq \mu_{\tilde{\tilde{G}}_{l}}^{\star}\left(h_{l}\right), \\
\bar{u}_{l} \sim N\left(\sum_{j=1}^{n}\left\{m_{l j}-L^{\star}\left(h_{l}\right) \alpha_{l j}\right\} x_{j}, \sum_{j=1}^{n} \sigma_{l j}^{2} x_{j}^{2}\right) .
\end{gathered}
$$

Since $P\left(\omega \mid u_{l}(\omega) \leq f_{l}\right)$ is transformed into

$$
\begin{gathered}
P\left(\omega \mid \frac{u_{l}(\omega)-\sum_{j=1}^{n}\left\{m_{l j}-L^{\star}\left(h_{l}\right) \alpha_{l j}\right\} x_{j}}{\sqrt{\sum_{j=1}^{n} \sigma_{l j}^{2} x_{j}^{2}}}\right. \\
\left.\leq \frac{f_{l}-\sum_{j=1}^{n}\left\{m_{l j}-L^{\star}\left(h_{l}\right) \alpha_{l j}\right\} x_{j}}{\sqrt{\sum_{j=1}^{n} \sigma_{l j}^{2} x_{j}^{2}}}\right),
\end{gathered}
$$

in consideration of

$$
\frac{\bar{u}_{l}-\sum_{j=1}^{n}\left\{m_{l j}-L^{\star}\left(h_{l}\right) \alpha_{l j}\right\} x_{j}}{\sqrt{\sum_{j=1}^{n} \sigma_{l j}^{2} x_{j}^{2}}} \sim N(0,1),
$$

(16) is equivalently transformed as

$$
\Phi\left(\frac{f_{l}-\sum_{j=1}^{n}\left\{m_{l j}-L^{\star}\left(h_{l}\right) \alpha_{l j}\right\} x_{j}}{\sqrt{\sum_{j=1}^{n} \sigma_{l j}^{2} x_{j}^{2}}}\right) \geq \mu_{\tilde{G}_{l}}^{\star}\left(h_{l}\right),
$$

where $\Phi$ is a probability distribution function of the standard Gaussian random variable $N(0,1)$.

From the monotone increasingness of $\Phi,(17)$ is rewritten as

$$
\sum_{j=1}^{n}\left\{m_{l j}-L^{\star}\left(h_{l}\right) \alpha_{l j}\right\} x_{j}+\Phi^{-1}\left(\mu_{\tilde{G}_{l}}^{\star}\left(h_{l}\right)\right) \sqrt{\sum_{j=1}^{n} \sigma_{l j}^{2} x_{j}^{2}} \leq f_{l},
$$

where $\Phi^{-1}$ is the inverse function of $\Phi$.

In this way, the augmented minimax problem (13) is equivalently transformed into

$$
\begin{aligned}
\operatorname{minimize} & \max _{l=1, \ldots, k}\left\{\hat{h}_{l}-h_{l}+\rho \sum_{l=1}^{k}\left(\hat{h}_{l}-h_{l}\right)\right\} \\
\text { subject to } & \sum_{j=1}^{n}\left\{m_{l j}-L^{\star}\left(h_{l}\right) \alpha_{l j}\right\} x_{j} \\
& +\Phi^{-1}\left(\mu_{\tilde{G}_{l}}^{\star}\left(h_{l}\right)\right) \sqrt{\sum_{j=1}^{n} \sigma_{l j}^{2} x_{j}^{2}} \leq f_{l}, \\
& A \boldsymbol{x} \leq \boldsymbol{b}, \boldsymbol{x} \geq \mathbf{0}
\end{aligned}
$$

Observing that (19) is an ordinary nonconvex nonlinear programming problem, it is possible to employ particle swarm optimization for nonlinear programming (PSONLP) [22] for obtaining an approximate solution.

Following the above discussions, we can now construct an interactive algorithm for deriving the satisficing solution for the DM from among the Pareto optimal solution set.

\section{Interactive fuzzy satisficing method}

Step 1: Ask the DM to specify the target values $f_{l}, l=$ $1, \ldots, k$, and determine the membership functions $\mu_{\tilde{G}_{l}}, l=1, \ldots, k$.

Step 2: Set the initial reference possibility levels at $1 \mathrm{~s}$, which can be viewed as the ideal values, i.e., $\hat{h}_{l}=$ $1, l=1, \ldots, k$.

Step 3: For the current reference possibility levels $\hat{h}_{l}$, $l=1, \ldots, k$, solve the corresponding augmented minimax problem (19).

Step 4: The DM is supplied with the corresponding Pareto optimal solution $\boldsymbol{x}^{*}$. If the DM is satisfied with the current objective function values $h_{l}^{*}, l=1, \ldots, k$, then stop. Otherwise, ask the DM to update the reference possibility levels $\hat{h}_{l}$, $l=1, \ldots, k$ by considering the current values of objective functions $h_{l}, l=1, \ldots, k$, and return to step 3.

It should be noted for the DM that any improvement of one objective function value can be achieved only at the expense of at least one of the other objective function values for the fixed target values $f_{l}, l=1, \ldots, k$.

\section{Numerical Example}

In order to demonstrate the feasibility and efficiency of the proposed interactive fuzzy satisficing method, as a numerical example of (2), consider the two-objective random fuzzy linear programming problem formulated as:

$$
\left.\begin{array}{ll}
\operatorname{minimize} & \overline{\tilde{C}}_{1} \boldsymbol{x} \\
\text { minimize } & \tilde{\boldsymbol{C}}_{2} \boldsymbol{x} \\
\text { subject to } & \boldsymbol{a}_{1} \boldsymbol{x} \leq b_{1} \\
& \boldsymbol{a}_{2} \boldsymbol{x} \leq b_{2} \\
& \boldsymbol{a}_{3} \boldsymbol{x} \leq b_{3} \\
& \boldsymbol{a}_{4} \boldsymbol{x} \leq b_{4} \\
& \boldsymbol{x}=\left(x_{1}, x_{2}, x_{3}, x_{4}, x_{5}, x_{6}\right)^{T} \geq \mathbf{0} .
\end{array}\right\}
$$

Table 1 shows values of coefficients of constraints $\boldsymbol{a}_{i}$, $i=1,2,3,4$ and $b_{i}, i=1,2,3,4$ and Table 2 shows values of parameters of random fuzzy variables $m_{l j}, \alpha_{l j}$ and $\sigma_{l j}^{2}, l=1,2, j=1,2,3,4,5,6$, where triangular fuzzy numbers are assumed for $\mu_{\tilde{M}_{l j}}(\tau)$. 
Table 1. Values of coefficients in constraints

\begin{tabular}{cccccccc}
\hline & $a_{i 1}$ & $a_{i 2}$ & $a_{i 3}$ & $a_{i 4}$ & $a_{i 5}$ & $a_{i 6}$ & $\boldsymbol{b}$ \\
\hline $\boldsymbol{a}_{1}$ & 1.00 & 3.00 & 4.00 & 2.00 & 5.00 & 2.00 & 120 \\
\hline $\boldsymbol{a}_{2}$ & 2.00 & 4.00 & 5.00 & 1.00 & 9.00 & 8.00 & 250 \\
\hline $\boldsymbol{a}_{3}$ & 5.00 & 2.00 & 1.00 & 4.00 & 5.00 & 8.00 & 400 \\
\hline $\boldsymbol{a}_{4}$ & 3.00 & 8.00 & 4.00 & 7.00 & 8.00 & 6.00 & 600 \\
\hline
\end{tabular}

Table 2. Values of $m_{l j}, \alpha_{l j}$ and $\sigma_{l j}^{2}$.

\begin{tabular}{ccccccc}
\hline & $\tilde{c}_{l 1}$ & $\tilde{c}_{l 2}$ & $\tilde{c}_{l 3}$ & $\tilde{c}_{l 4}$ & $\tilde{c}_{l 5}$ & $\tilde{c}_{l 6}$ \\
\hline$m_{1 j}$ & -7.30 & -4.50 & -5.00 & -3.30 & -5.50 & -3.8 \\
\hline$m_{2 j}$ & 2.50 & 4.20 & 3.00 & 4.90 & 2.50 & 4.30 \\
\hline$\alpha_{1 j}$ & 0.70 & 0.50 & 0.80 & 0.40 & 0.70 & 0.60 \\
\hline$\alpha_{2 j}$ & 0.90 & 0.80 & 0.70 & 0.40 & 0.60 & 0.50 \\
\hline$\sigma_{1 j}^{2}$ & 1.40 & 1.00 & 1.10 & 1.20 & 1.10 & 0.90 \\
\hline$\sigma_{2 j}^{2}$ & 1.00 & 1.00 & 1.20 & 1.20 & 0.80 & 1.00 \\
\hline
\end{tabular}

Through the use of this numerical example, it is now appropriate to illustrate the proposed interactive fuzzy satisficing method.

The parameter values of particle swarm optimization for nonlinear programming (PSONLP) are set as swarm size $N=50$, maximal search generation number $T_{\max }=$ $3000, c_{1}=2.0, c_{2}=2.0, w^{0}=1.2$ and $w^{T_{\max }}=0.1$.

Suppose that the DM sets the target values $f_{l}, l=$ 1,2 as $f_{1}=-300, f_{2}=100$, and determines the linear membership functions $\mu_{\tilde{G}_{l}}, l=1,2$.

For the initial reference possibility levels $\hat{h}_{l}=1, l=$ 1,2 , the corresponding augmented minimax problem is solved, and the obtained result is shown at the column labeled "1st" in Table 3. DM is not satisfied with these values of objective functions, and the DM updates the reference probability levels as $(1.0,0.7)$ for improving the values of objective functions $h_{1}$ at the expense of $h_{2}$. For the updated reference fractile levels, the corresponding augmented minimax problem is solved, and the obtained result is shown at the column labeled "2nd" in Table 3.

A similar procedure continues until the DM is satisfied with the values of objective functions. In this example, we assume that the satisficing solution for the DM is derived in the third interaction.

Table 3. Interaction process.

\begin{tabular}{cccc}
\hline Interaction & 1st & 2nd & 3rd \\
\hline$\hat{h}_{1}$ & 1.0 & 1.0 & 0.8 \\
\hline$\hat{h}_{2}$ & 1.0 & 0.7 & 0.7 \\
\hline$x_{1}$ & 26.52 & 21.22 & 23.78 \\
\hline$x_{2}$ & 2.02 & 4.20 & 3.09 \\
\hline$x_{3}$ & 0.45 & 11.31 & 2.03 \\
\hline$x_{4}$ & 4.39 & 0.82 & 2.95 \\
\hline$x_{5}$ & 14.65 & 5.39 & 6.00 \\
\hline$x_{6}$ & 0.08 & 1.62 & 1.51 \\
\hline$h_{1}$ & 0.64 & 0.74 & 0.68 \\
\hline$h_{2}$ & 0.64 & 0.58 & 0.62 \\
\hline
\end{tabular}

\section{Conclusions}

In this paper, random fuzzy multiobjective linear programming problems have been considered. For tackling the formulated problems, it has been assumed that the decision maker concerns about the probabilities that each of the objective function values is smaller than or equal to a certain target value. By introducing the fuzzy goals of the decision maker for the probabilities and assuming that the decision maker is willing to maximize the degrees of possibility with respect to the attained probability, an interactive fuzzy satisficing method has been presented for deriving a satisficing solution for the decision maker by updating the reference possibility levels. It was shown that all of the problems to be solved in the proposed interactive fuzzy satisficing method can be solved through particle swarm optimization for nonlinear programming (PSONLP). An illustrative numerical example was provided to demonstrate the feasibility of the proposed method. However, further computational experiences should be carried out for several types of numerical examples. From such experiences the proposed computational method must be revised. Applications of the proposed method to the real world decision making situations will be required in the near future. Extensions to other stochastic programming models will be considered elsewhere. Also extensions to integer programming problems involving random fuzzy variable coefficients will be required in the near future.

\section{REFERENCES}

[1] J.R. Birge, F. Louveaux, Introduction to Stochastic Programming, Springer, London, 1997.

[2] P. Kall, J. Mayer, Stochastic Linear Programming: Models, Theory, and Computation, 2nd Edition, Springer, New York, 2011.

[3] I. M. Stancu-Minasian, Stochastic Programming with Multiple Objective Functions, D. Reidel Publishing Company, Dordrecht, 1984.

[4] I. M. Stancu-Minasian, Overview of different approaches for solving stochastic programming problems with multiple objective functions, In R. Slowinski, J. Teghem, (Eds.), Stochastic Versus Fuzzy Approaches to Multiobjective Mathematical Programming under Uncertainty, Kulwer Academic Publishers, Dordrecht/Boston/London, 71-101, 1990.

[5] M. Sakawa, Fuzzy Sets and Interactive Multiobjective Optimization, Plenum Press, New York, 1993.

[6] H.-J. Zimmermann, Fuzzy programming and linear programming with several objective functions, Fuzzy Sets and Systems, Vol. 1, No. 1, pp. 45-55, 1978.

[7] H.-J. Zimmermann, Fuzzy Sets, Decision-Making and Expert Systems, Kluwer Academic Publishers, Boston, 1987.

[8] G.B. Dantzig, Linear programming under uncertainty, Management Science, Vol. 1, No. 3-4, pp.197-206, 1955.

[9] A. Charnes, W.W. Cooper, Chance constrained programming, Management Science, Vol. 6, No. 1, pp. 7379, 1959. 
[10] M. Sakawa, H. Yano, Interactive fuzzy satisficing method using augmented minimax problems and its application to environmental systems, IEEE Transactions on Systems, Man and Cybernetics, Vol. SMC-15, No. 6, pp. 720-729, 1985.

[11] M. Sakawa, K. Kato, Interactive fuzzy multi-objective stochastic linear programming, In C. Kahraman (Ed.), Fuzzy Multi-Criteria Decision Making - Theory and Applications with Recent Developments -, Springer, New York, 375-408, 2008.

[12] M. Sakawa, K. Kato, I. Nishizaki, An interactive fuzzy satisficing method for multiobjective stochastic linear programming problems through an expectation model, European Journal of Operational Research, Vol. 145, No. 3, pp. 665-672, 2003.

[13] M. Sakawa, K. Kato, An interactive fuzzy satisficing method for multiobjective stochastic linear programming problems using chance constrained conditions, Journal of Multi-Criteria Decision Analysis, Vol.11, No. 3, pp. 125-137, 2002.

[14] M. Sakawa, K. Kato, H. Katagiri, An interactive fuzzy satisficing method for multiobjective linear programming problems with random variable coefficients through a probability maximization model, Fuzzy Sets and Systems, Vol. 146, No. 2, pp. 205-220, 2004.

[15] H. Kwakernaak, Fuzzy random variables - I. definitions and theorems, Information Sciences, Vol. 15, No. 1, pp. 1-29, 1978.
[16] M. L. Puri, D. A. Ralescu, Fuzzy random variables, Journal of Mathematical Analysis and Applications, Vol. 114, No. 2, pp. 409-422, 1986.

[17] G.-Y. Wang, Z. Qiao, Fuzzy programming with fuzzy random variable coefficients, Fuzzy Sets and Systems, Vol. 57, No. 3, pp. 295-311, 1993.

[18] Z. Qiao, Y. Zhang, G.-Y. Wang, On fuzzy random linear programming, Fuzzy Sets and Systems, Vol. 65, No. 1, pp. 31-49, 1994.

[19] B. Liu, Random fuzzy dependent-chance programming and its hybrid intelligent algorithm, Information Sciences, Vol. 141, No. 3-4, pp. 259-271, 2002.

[20] M. Sakawa, I. Nishizaki, H. Katagiri, Fuzzy Stochastic Multiobjective Programming, Springer, New York, 2011.

[21] S. Nahmias, Fuzzy variables, Fuzzy Sets and Systems, Vol. 1, No. 2, pp. 97-110, 1978.

[22] T. Matsui, M. Sakawa, K. Kato, T. Uno, K. Tamada, Particle swarm optimization for interactive fuzzy multiobjective nonlinear programming, Scientiae Mathematicae Japonicae, Vol. 68, No. 1, pp. 103-115, 2008. 